\title{
The 3D solar corona Cycle 24 rising phase from SDO/AIA tomography
}

\author{
Federico A. Nuevo ${ }^{1,2}$, Alberto M. Vásquez ${ }^{1,2}$, Richard A. Frazin ${ }^{3}$, \\ Zhenguang Huang ${ }^{3}$, and Ward B. Manchester IV $^{3}$ \\ ${ }^{1}$ Instituto de Astronomía y Física del Espacio (CONICET-UBA), \\ CC 67 - Suc 28, (C1428ZAA) Ciudad de Buenos Aires, Argentina \\ email: federico@iafe.uba.ar \\ ${ }^{2}$ Facultad de Cs. Exactas y Naturales, Universidad de Buenos Aires, Argentina \\ ${ }^{3}$ Department of Atmospheric, Oceanic and Space Sciences, \\ University of Michigan, Ann Arbor, MI 48109, USA
}

\begin{abstract}
We recently extended the differential emission measure tomography (DEMT) technique to be applied to the six iron bands of the Atmospheric Imaging Assembly (AIA) instrument aboard the Solar Dynamics Observatory (SDO). DEMT products are the 3D reconstruction of the coronal emissivity in the instrument's bands, and the 3D distribution of the local differential emission measure, in the height range 1.0 to $1.25 \mathrm{R}_{\odot}$. We show here derived maps of the electron density and temperature of the inner solar corona during the rising phase of solar Cycle 24. We discuss the distribution of our results in the context of open/closed magnetic regions, as derived from a global potential field source surface (PFSS) model of the same period. We also compare the results derived with SDO/AIA to those derived with the Extreme UltraViolet Imager (EUVI) instrument aboard the Solar TErrestrial RElations Observatory (STEREO).
\end{abstract}

Keywords. Sun: corona, Sun: fundamental parameters, methods: data analysis

\section{Introduction}

The DEMT technique is described in detail in Frazin et al. (2009). We briefly summarize here its key elements, focusing on the determination of the local differential emission measure (LDEM), pertaining only to the plasma contained within each tomographic grid cell (see below). The inner corona $\left(1.0-1.25 \mathrm{R}_{\odot}\right)$ is discretized on a $25 \times 90 \times 180$ (radial $\times$ latitudinal $\times$ longitudinal) spherical computational grid. A time series of EUV images covering a full solar rotation is tomographically inverted, for each band $k$ separately, to obtain the filter band emissivity (FBE), $\zeta_{i}^{(k)}$, at every tomographic cell $i$. The tomographic inversion assumes a static corona, so that solar dynamics occurring in the Sun produce artifacts in the reconstructions. Such artifacts include smearing and negative values of the reconstructed FBEs, or zero when the solution is constrained to positive values. These are called zero density artifacts (ZDAs).

The FBE values at each cell are then used to perform the LDEM analysis. In this work we parametrize the LDEM as a combination of one or two normal distributions, $\xi_{i}(T)=\mathcal{N}\left(T ; \lambda_{i}=\left[T_{0}, \sigma_{T}, a\right]_{i}\right.$ ) (in a similar way to Aschwanden \& Boerner 2011), to treat three EUVI and four AIA bands (see Section 2), respectively. In the case of AIA, one of the normal distributions has its centroid fixed at the maximum sensitivity temperature of the $335 \AA$ band. To find $\lambda_{i}$ we minimize the sum of the quadratic deviations between the synthesized and tomographic FBEs: $\Phi\left(\lambda_{i}\right)=\sum_{k=1}^{K}\left[\zeta_{i}^{(k)}-\int d T \xi\left(T ; \lambda_{i}\right) Q_{k}(T)\right]^{2}$, where $Q_{k}(T)$ is the temperature response of the $k$-th band. Fig. 1 shows the temperature responses of the coronal bands we used, for both the STEREO/EUVI and the 
SDO/AIA instruments, computed using CHIANTI v6.0.1, assuming the Feldman et al. (1992) abundance set, and the Bryans et al. (2009) ionization equilibrium calculations. Using the LDEM we derive plasma electron parameters at each tomographic grid cell $i$,

$$
\begin{aligned}
N_{e, i}^{2} & =\int d T \xi_{i}(T), \\
T_{m, i} & =\left(1 / N_{e, i}^{2}\right) \int d T \xi_{i}(T) T, \\
W_{T, i}^{2} & =\left(1 / N_{e, i}^{2}\right) \int d T \xi_{i}(T)\left(T-T_{m, i}\right)^{2},
\end{aligned}
$$

and quantify the success of the LDEM in reproducing the $K$ tomographic FBEs as,

$$
\chi_{i}^{2}=\frac{1}{K} \sum_{k=1}^{K}\left(1-\zeta_{i}^{(k, \text { synth })} / \zeta_{i}^{(k)}\right)^{2},
$$

where the $\zeta_{i}^{(k, \text { synth })}$ is given by the $k$-th temperature integral within the functional $\Phi$ defined above. We consider a cell to have a non-satisfactory-fit (NSF) of the parametrized LDEM when $\chi^{2}>5 \times 10^{-2}$, or a mean quadratic deviation of order $20 \%$ between the tomographic and synthesized FBEs.

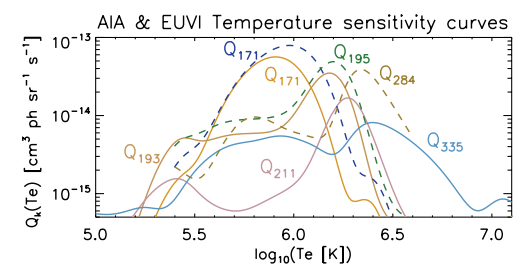

Figure 1. Temperature responses of the SDO/AIA (solid) and STEREO/EUVI-B (dashed).

Figures in colour can be found in the online version.

\section{Results}

We performed two DEMT analyses of the period CR 2106 (2011, 20 January through 16 February), one using STEREO/EUVI data, and another one using SDO/AIA. For STEREO/EUVI we obtained the 3D FBE distribution for its three coronal bands (171, 195, and $284 \AA$ ), while for SDO/AIA we did the same for its four stronger bands (171, 193,211 , and $335 \AA$ ). Using each set of FBE tomographic reconstructions separately, we derived the LDEM moments from Eqs. (1.1)-(1.3).

Fig. 2 shows the DEMT results and $\chi^{2}$ at $1.075 \mathrm{R}_{\odot}$, with similar maps obtained at all 25 tomographic grid height bins. Using a PFSS extrapolation of the same period based on GONG data, we overplot magnetic- strength $B$ contour levels (thin black and white curves for negative and positive polarity), as well as the magnetically open/closed region boundaries (thick black curves). The PFSS model is computed using the finite-difference iterative solver FDIPS by Tóth et al. (2011), on a $150 \times 180 \times 360$ spherical grid, covering 1.0 to $2.5 \mathrm{R}_{\odot}$. While the morphology of the corona is clearly more complex compared to solar minimum, there is an overall good agreement between the magnetic-PFSSM and the density/temperature-DEMT structures.

The differences in the $N_{e}, T_{m}$ and $W_{T}$ maps obtained from both data sets are mainly due to the inclusion of AIA $335 \AA$ band, and also to differences between the temperature 

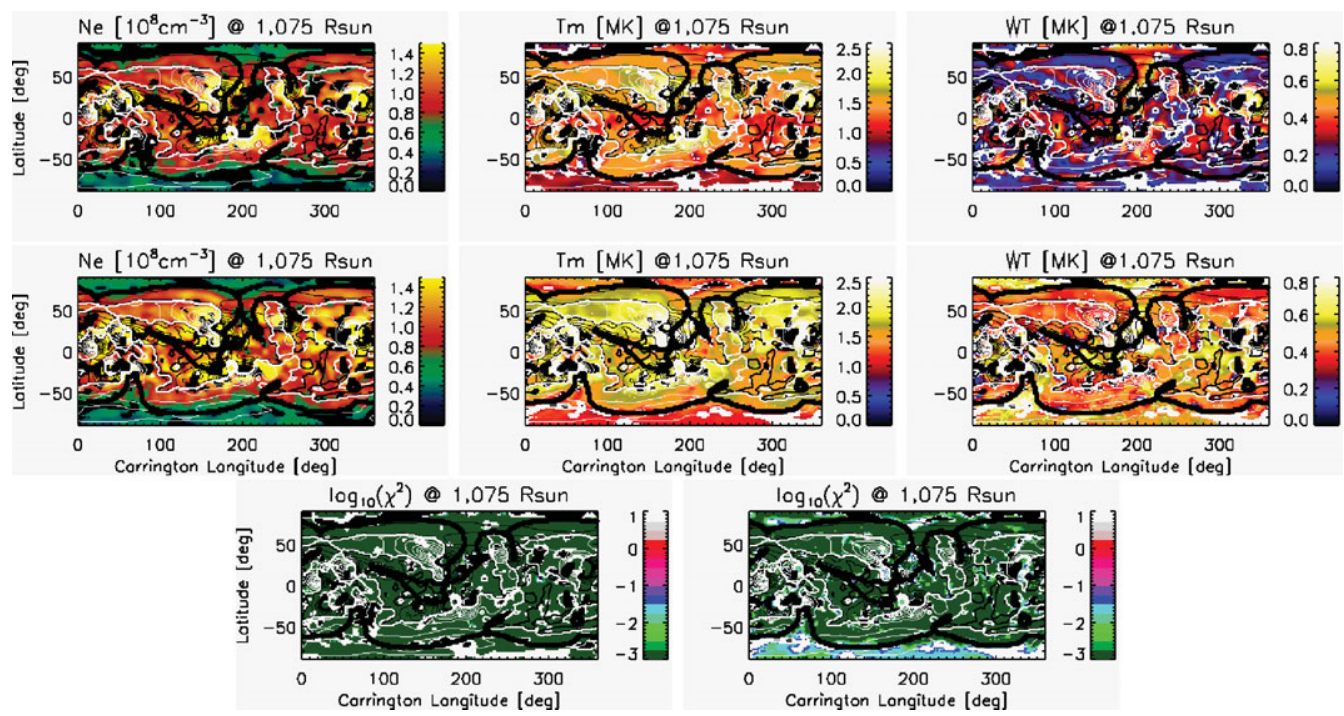

Figure 2. Top panels: $N_{e}, T_{m}$ and $W_{T}$ map from STEREO/EUVI data at $1.075 \mathrm{R}_{\odot}$ with a single-normal parametrization. Middle panels: $N_{e}, T_{m}$ and $W_{T}$ map from SDO/AIA data at $1.075 \mathrm{R}_{\odot}$ with a double-normal parametrization. Bottom panels: $\chi^{2}$ maps for STEREO/EUVI result (left) and SDO/AIA results (right). ZDAs and NSFs are indicated as black and white cells, respectively, in the $T_{m}, W_{T}$, and $\chi^{2}$ maps, and as black cells in the $N_{e}$ maps.

responses of the other three AIA bands with those of the EUVI instrument. Fig. 3 shows scatter plots and histograms comparing the DEMT results obtained with EUVI and AIA. The AIA results show systematically larger values, with $N_{e}, T_{m}$ and $W_{T}$ median values increasing by 9,16 , and $71 \%$, respectively, when compared to the EUVI results.
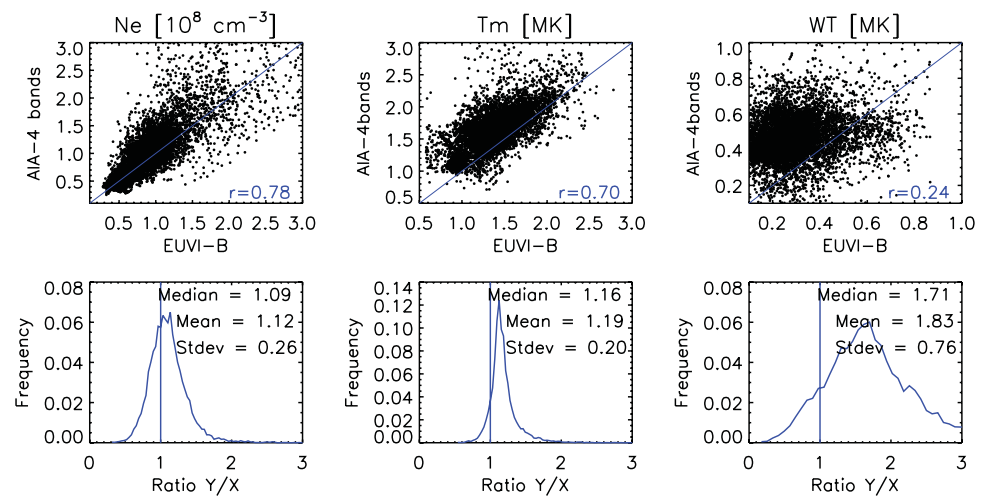

Figure 3. Comparison of LDEM results from EUVI and from AIA, at $1.075 \mathrm{R}_{\odot}$.

Fig. 4 shows statistics comparing the AIA DEMT results in open vs. closed regions (as determined from the PFSS model) at $1.075 \mathrm{R}_{\odot}$. The AIA DEMT results show that the streamer plasma is about $100 \%$ more dense and $50 \%$ hotter than in open regions. Also, when compared to EUVI DEMT CR-2077 and CR-2068 Solar Minimum results (see Vásquez et al. 2012, in this volume), the CR-2106 EUVI DEMT results indicate the streamer region is $\sim 10 \%$ more dense and and $\sim 20 \%$ hotter.

Fig. 5 shows the dependence with height of the AIA DEMT results for selected streamer regions, averaged over the range of latitudes and longitudes specified in each plot. We 

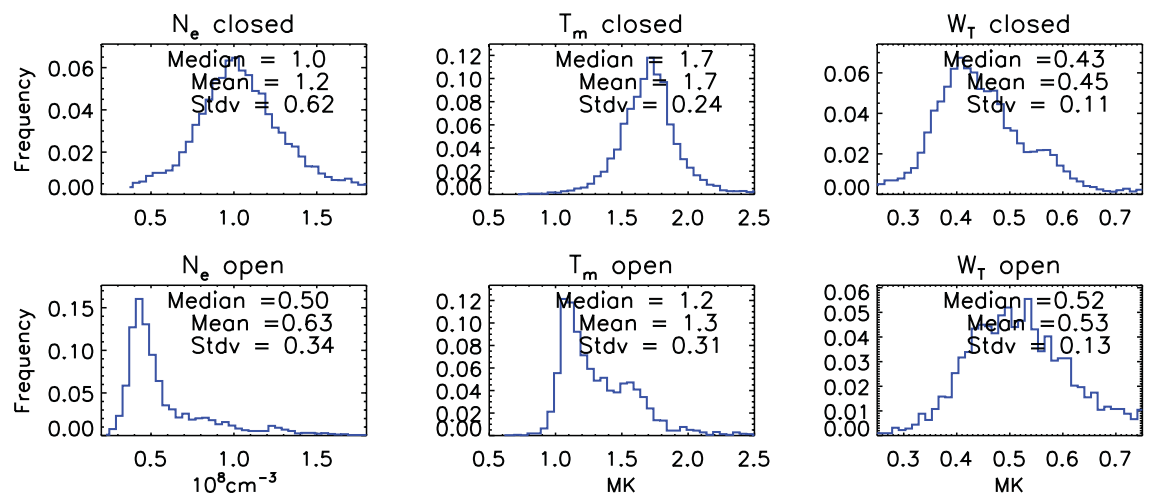

Figure 4. Open-vs-Closed statistics of the LDEM moments at $1.075 \mathrm{R}_{\odot}$.

apply hydrostatic fits to the LDEM $N_{e}(r)$ data, and find the electron temperature $T_{e}$,fit from the fit scale height, assuming $T_{e}=T_{\mathrm{H}}$. Taking then the LDEM $<T_{m}>$ as a measure of the true $T_{e}$, it follows $\frac{T_{\mathrm{H}}}{\left\langle T_{m}\right\rangle} \approx 1+2\left(\frac{\left.T_{e, \text { fit }}-<T_{m}\right\rangle}{\left\langle T_{m}\right\rangle}\right)$ (neglecting the He abundance). The CR-2106 streamer results show consistency with hydrostatic equilibrium, as the correlation coefficients are high, both in active and quiet zones. The fact that in those regions $T_{e, \text { fit }}<<T_{m}>$, may then be indicative of $T_{e}>T_{\mathrm{H}}$ (Vásquez et al. 2011).
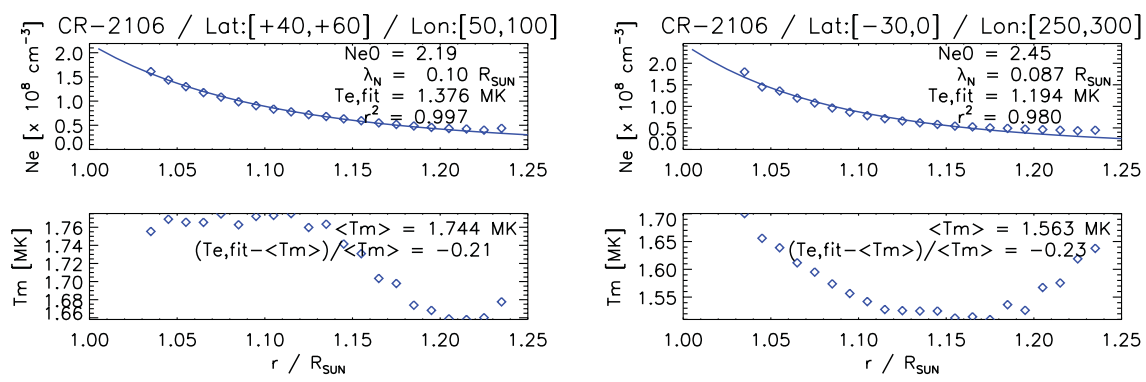

Figure 5. Average dependence with height of LDEM moments for selected streamer regions, and hydrostatic fits to the LDEM $N_{e}(r)$ data.

In a future publication we will develop a study of EUVI and AIA inversions of simulated DEM data to learn how to interpret the differences arising from the DEMT analysis based on both instruments.

\section{Acknowledgements}

F.A.N. and A.M.V. acknowledge CLAF and IAU for their support to attend IAU Symposium 286 in Mendoza, Argentina.

\section{References}

Aschwanden, M. \& Boerner, P. 2011, ApJ, 732, 81-95

Bryans, P., Landi, E., \& Savin, W. 2009, ApJ, 691, 1540-1559

Feldman, U., Mandelbaum, P., Seely, J. L., Doschek, G. A., \& Gursky, H. 1992, ApJ, 81, 387-408

Frazin, R. A., Vásquez, A. M., \& Kamalabadi, F. 2009, ApJ, 701, 547-560

Tóth, G., van der Holst, B., \& Huang,Z. 2011, ApJ, 732, 102-108

Vásquez, A. M., Huang, Z., Manchester IV, W. B., \& Frazin, R. A. 2011, Sol. Phys., 274, 259-284

Vásquez, A. M., Frazin, R. A., Huang, Z., Manchester IV, W. B., \& Shearer, P. 2012, this volume 